\title{
The influence of planetary engulfment on stellar rotation in metal-poor main-sequence stars
}

\author{
A. Oetjens ${ }^{2,1}$, L. Carone ${ }^{1}$, M. Bergemann ${ }^{1}$, and A. Serenelli ${ }^{3,4}$ \\ ${ }^{1}$ Max-Planck-Institute for Astronomy, Königstuhl 17, 69117 Heidelberg, Germany \\ e-mail: oetjens@mpia-hd.mpg.de \\ 2 Ruprecht-Karls-Universität, Grabengasse 1, 69117 Heidelberg, Germany \\ 3 Institute of Space Sciences (ICE, CSIC) Campus UAB, Carrer de Can Magrans, s/n, 08193 Bellaterra, Spain \\ 4 Institut d'Estudis Espacials de Catalunya (IEEC), C/Gran Capita, 2-4, 08034 Barcelona, Spain
}

Received 14 June 2020 / Accepted 7 September 2020

\begin{abstract}
Context. The method of gyrochronology relates the age of its star to its rotation period. However, recent evidence of deviations from gyrochronology relations has been reported in the literature.

Aims. We study the influence of tidal interaction between a star and its companion on the rotation velocity of the star to explain peculiar stellar rotation velocities.

Methods. We followed the interaction of a star and its planet using a comprehensive numerical framework that combines tidal friction, magnetic braking, planet migration, and detailed stellar evolution models from the GARSTEC grid. We focus on close-in companions from 1 to $20 M_{\text {Jup }}$ orbiting low-mass $\left(0.8-1 M_{\odot}\right)$ main-sequence stars with a broad metallicity of $[\mathrm{Fe} / \mathrm{H}]=-1$ up to solar.

Results. Our simulations suggest that the dynamical interaction between a star and its companion can have different outcomes that depend on the initial semi-major axis and the mass of the planet, as well as on the mass and metallicity of its host star. In most cases, especially in the case of planet engulfment, we find a catastrophic increase in stellar rotation velocity from $1 \mathrm{kms}^{-1}$ to over $40 \mathrm{kms}^{-1}$ while the star is still on the main-sequence. The main prediction of our model is that low-mass main-sequence stars with abnormal rotation velocities should be more common at low-metallicity, as lower $[\mathrm{Fe} / \mathrm{H}]$ favours faster planet engulfment, based on the assumption that the occurrence rate of close-in massive planets is similar at all metallicities.

Conclusions. Our scenario explains peculiar rotation velocities of low-mass main-sequence stars by the tidal interaction between the star and its companion. Current observational samples are too narrow and incomplete, and, thus, they are not sufficient for our model to be tested.
\end{abstract}

Key words. planet-star interactions - stars: rotation - stars: low-mass

\section{Introduction}

The past decade has brought revolutionary advances in spacebased astronomical instrumentation. Space missions, such as Kepler, CoRoT, and TESS, have enabled astronomers to collect extended time series of stellar surface brightness observations. These surveys have provided an unprecedented view onto the population statistics and physical properties of exoplanets (e.g. Buchhave et al. 2012; Fressin et al. 2013; Weiss et al. 2018; Zhou et al. 2019). They have also triggered a massive application of asteroseismology techniques to the photometric data with the aim of constraining the interior structure of stars (e.g. Degroote et al. 2010; Beck et al. 2012; Chaplin \& Miglio 2013; Chaplin et al. 2014; Metcalfe et al. 2014; Silva Aguirre et al. 2015).

A combination of data and models enables studies of a coupled physical and dynamical evolution of a planet and its host star (e.g. Van Eylen et al. 2018; Beck et al. 2018). The star-planet interaction has two major consequences for the structure of the star: a change in the surface chemical composition (e.g. Meléndez et al. 2012; Spina et al. 2015; Petrovich \& Muñoz 2017; Veras \& Fuller 2019) and a change in the stellar rotation period (Carone \& Pätzold 2007; Cassan et al. 2012; Fleming et al. 2019). Both effects are still poorly explored with the available models and, in particular, it is still not well understood whether (and how) the angular momentum of a star changes under the influence of the companion. This problem is the focus of our work.

The rotation of a single main-sequence (MS) star has canonically been assumed to follow the empirical (Skumanich 1972) relation, which relates the rotation rate of a star $\Omega$ to its age, $\Omega \sim t^{-0.5}$. This relationship was applied to stars in open clusters (e.g. Schatzman 1962; Bouvier et al. 1997; Barnes 2003; Meibom et al. 2015) and it forms the basis of standard gyrochronology relationships (e.g. Barnes 2007; Angus et al. 2015, 2019; Claytor et al. 2020). In this model, angular momentum is removed by magnetised stellar winds through the phenomenon known as magnetic braking (e.g. Parker 1958; Weber \& Davis 1967; Bouvier et al. 1997; Barnes 2003).

However, recent studies of ages derived by different methods raised controversy over the universal applicability of the Skumanich law. Deviations from this law were observed for a fraction of normal MS stars (e.g. Nielsen et al. 2015; Sahlholdt et al. 2019). This can be accommodated within two scenarios: the change in stellar magnetic field configuration (e.g. van Saders et al. 2016; Metcalfe et al. 2019) or angular momentum transfer via tidal friction from a close companion (e.g Fleming et al. 2019; Santos et al. 2019). 

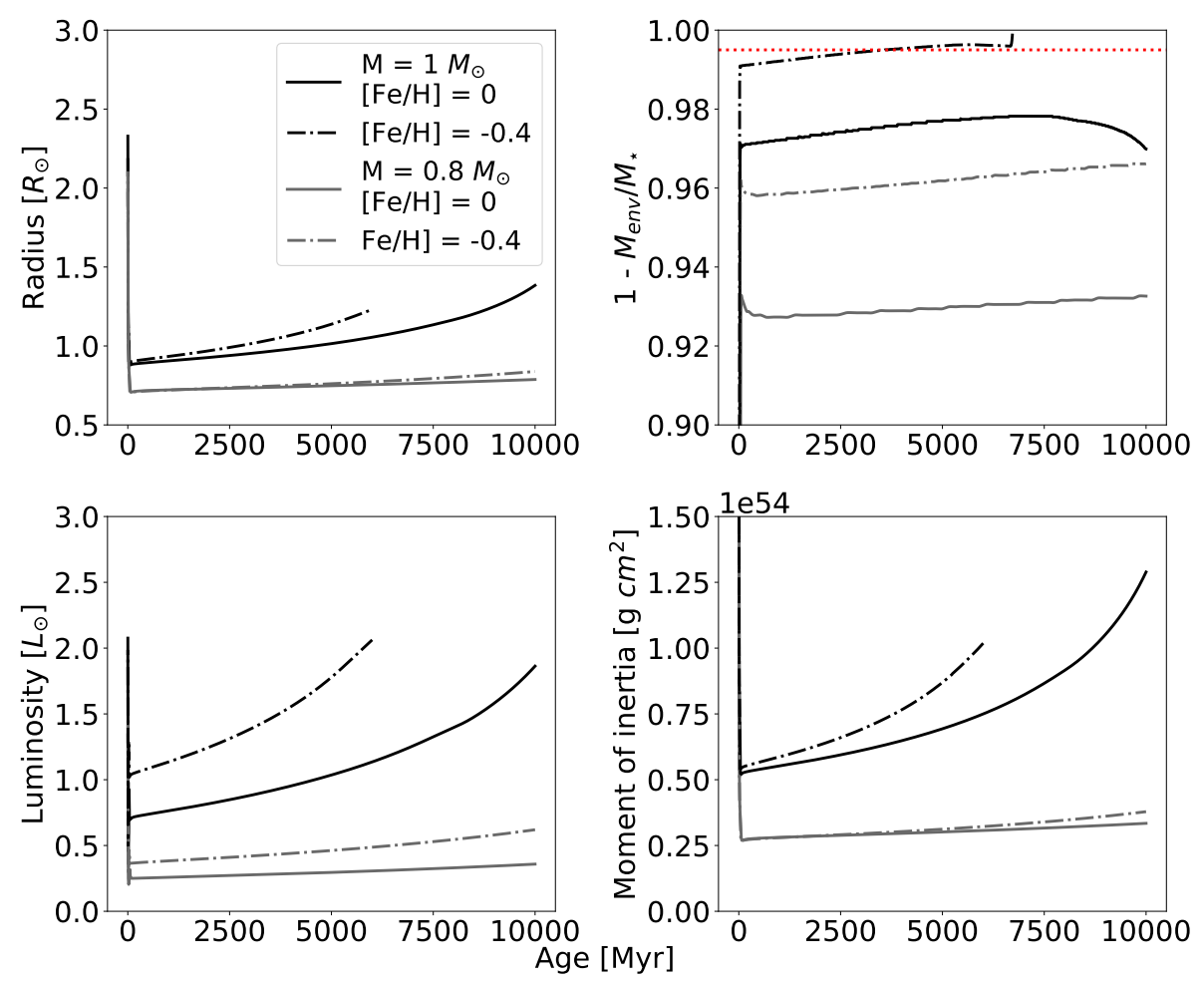

Fig. 1. Profiles of different MS stellar parameters against the age of a star for four representative evolutionary tracks computed with the mass of 0.8 and $1 M_{\odot}$ and $[\mathrm{Fe} / \mathrm{H}]=-0.4$ and 0 (solar metallicity). The top-right plot shows the mass of the convective envelope with the limit of $M_{\mathrm{env}} / M_{\odot} \approx 0.005$, as indicated by the dotted red line.

Recently, Amard \& Matt (2020) applied the magnetic braking formalism of Matt et al. (2015) to metal-poor stars, which takes the Rossby number (and hence the convective turnover timescale) into account. The authors predict deviations from the gyrochronology picture for stars (in a mass range of $1-1.5 M_{\odot}$ ) of lower metallicity $([\mathrm{Fe} / \mathrm{H}] \lesssim-0.5)$ due to a lower efficiency in magnetic braking.

In this paper, we focus on changes of stellar rotation ${ }^{1}$ caused by the tidal interaction of a star with a close planetary or lowmass brown dwarf companion using methods and models by Rasio et al. (1996), Bouvier et al. (1997), and Carone \& Pätzold (2007), combining them with state-of-the-art stellar structure models (Serenelli et al. 2013, 2017). We quantify the spin-up rate as a function of the mass and metallicity of a star, as well as the orbit and mass of the companion

The paper is organised as follows. The model we used to calculate the dynamical interaction between a star and its planet is presented in Sect. 2. The results of our modelling are discussed in Sect. 3. We discuss the implications of our results in Sect. 4. We summarise our results and present further possible avenues of investigation in Sect. 5.

\section{Dynamical model for star-planet interaction}

We use the star-planet interaction formalism established by Carone \& Pätzold (2007), which combines tidal migration with magnetic braking, in order to quantify the impact of a tidally migrating massive companion with $1-20 M_{\text {Jup }}$ on the rotation rate of its host star. We mainly refer to the companion as a "massive planet" and we do not specifically differentiate between massive planets ( $<13 M_{\text {Jup }}$ for solar metallicity) and low-mass

\footnotetext{
1 We note that in tidal interaction literature it is also common to speak about "qspin" instead of rotation.
}

brown dwarfs ( $\geq 13 M_{\text {Jup }}$ for solar metallicity) $)^{2}$. We focus on stars in the MS evolutionary phase and apply the model to stars with masses in the range of $0.8-1 M_{\odot}$ and metallicities from $-1 \leq[\mathrm{Fe} / \mathrm{H}] \leq 0 \mathrm{dex}$, in order to check the influence of chemical composition on the tidal interaction. Only massive planets on very tight orbits with less than 0.03 AU can tidally migrate inwards and spin up their star within the MS lifetime (Carone \& Pätzold 2007; Jackson et al. 2009).

All quantities, which describe the global properties of a star as a function of its evolutionary stage - the mass $M_{\text {env }}$ and the radius $R_{\text {env }}$ of the convective envelope, stellar radius $R_{\star}$ and luminosity $L_{\star}-$ are taken from the Garstec stellar evolution models (Weiss \& Schlattl 2008; Serenelli et al. 2013, 2017). The grid of Garstec models covers the full parameter space of low-mass stars: mass in the range from 0.6 to $5 M_{\odot}$ and metallicity $[\mathrm{Fe} / \mathrm{H}]$ in the range from -2.5 to +0.6 in increments of 0.05 dex. This study focuses on the parameter space of 0.8 and $1 M_{\odot}$ and $[\mathrm{Fe} / \mathrm{H}]$ from -1 to 0 . The profiles of different stellar parameters for several evolutionary tracks are shown in Fig. 1.

\subsection{Gain of angular momentum through tidal friction}

We focus on the effect of the equilibrium tide on the star, which best describes tidal interactions of a companion with a low-mass star of $1 M_{\odot}$ and less (Fleming et al. 2019). Furthermore, we use the tidal friction formulation that was first established for binary stars by Zahn (1977) and expanded by Rasio et al. (1996) and Privitera et al. (2016) to extrasolar planets around MS stars, which allows us to self-consistently combine the efficiency of tidal migration with stellar structure. In this formulation, tidal dissipation occurs predominantly in the convective envelope of a star. The frictional loss of energy is then extracted from the orbit of the companion with the semi-major axis $a$ and planetary revolution rate $n$. We can safely assume a circular orbit because

\footnotetext{
2 http://w.astro.berkeley.edu/ basri/defineplanet/ IAU-WGEXSP.htm
} 
$e \approx 0$ is quickly achieved via efficient eccentricity damping for planet-mass objects on orbits with semi-major axis of $0.03 \mathrm{AU}$ and shorter (Dobbs-Dixon et al. 2004) $)^{3}$. The rate of orbital decay is given by:

$\left(\frac{\dot{a}}{a}\right)_{\text {tidal }}=\frac{f}{\tau} \frac{M_{\mathrm{env}}}{M_{\star}} q(1+q)\left(\frac{R_{\star}}{a}\right)^{8}$,

where $\tau$ is the convective turnover timescale, $M_{\mathrm{env}}$, is the mass of the convective envelope of a star at a given point in time, $M_{\star}$ and $R_{\star}$ are the total mass and radius of a star, and $q$ is the planetto-star mass ratio $q=M_{\mathrm{pl}} / M_{\star}$.

The numerical factor $f$ is given by

$f=\min \left[1,\left(\frac{P_{\mathrm{pl}}}{2 \tau}\right)^{2}\right]$

and it quantifies viscous dissipation of tidal energy throughout the convective zone. The $P_{\mathrm{pl}} / 2$ is the so-called tidal pumping frequency and accounts for the occurrence of two tidal bulges during one orbital revolution of the planetary companion around its star. If the convective turnover timescale, $\tau$, is short compared to the tidal pumping frequency, dissipation is very efficient and $f=1$. Otherwise, only those eddies with timescales shorter than $P_{\mathrm{pl}} / 2$ can contribute to the dissipation of energy and $f$ is smaller than 1 (Goldreich \& Keeley 1977; Rasio et al. 1996).

The convective eddy turnover timescale is calculated using the equation suitable for large eddies at the base of the convective zone (Rasio et al. 1996; Privitera et al. 2016):

$\tau=\left[\frac{M_{\mathrm{env}}\left(R_{\star}-R_{\mathrm{env}}\right)^{2}}{3 L_{\star}}\right]^{1 / 3}$,

where $L_{\star}$ is the luminosity of a star.

We note that in a substantial proportion of the literature on tidal interaction (see e.g. Jackson et al. 2009; Barnes 2015; Yee et al. 2020), instead of $\tau$, the tidal dissipation factor, $Q_{\star}$, or the modified tidal dissipation factor, $Q_{\star}^{\prime}=Q_{\star} / k_{2, \star}$, is used to quantify the efficiency of tidal interactions, where the latter also comprises the stellar Love number $k_{2, \star}$. To facilitate a comparison with other works, we also introduce a method of deriving the modified tidal dissipation factor, $Q_{\star}^{\prime}$, within the framework of this work via:

$Q_{\star}^{\prime}=\frac{3 G M_{\star}}{R_{\star}^{3} n} \frac{\tau}{f} \frac{M_{\star}}{M_{\mathrm{env}}}$.

For a comparison with other tidal interaction works, we further calculated the modified stellar tidal dissipation factor and derived $Q_{\star}^{\prime}=10^{7}$ for the systems that we studied. These $Q_{\star}^{\prime}$ values fit well into the range of $Q^{\prime}$ values used in the literature (see e.g. Barnes 2015; Heller 2019).

In tidal theory, many equations, such as the differential equation for the semi-major axis evolution description used in Carone \& Pätzold (2007), compare the orbital revolution rate, $n$, with the stellar rotation rate, $\Omega_{\star}$. The revolution rate $n$ is more informative in the framework of revolving and rotating bodies of unequal mass, where two bodies (one planetary mass and one stellar mass) orbit around the common centre of mass. However, the centre of mass lies inside the host star, such that we can assume, to the first order, that the planet revolves around the host star (see Fig. 2).

\footnotetext{
3 See also exoplanet.eu that shows $e \approx 0$ for the vast majority of hot Jupiters on tight orbits.
}

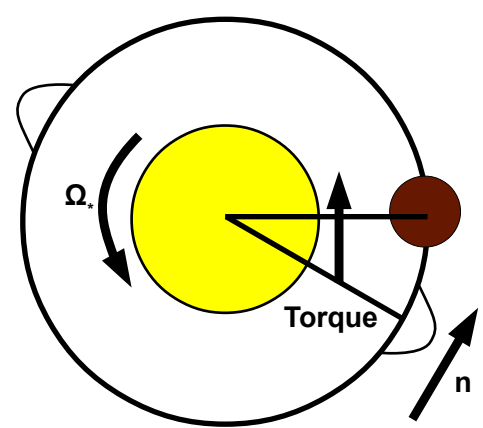

Fig. 2. Angular momentum transfer during planetary engulfment due to tidal friction. See also Fig. 1 in Carone \& Pätzold (2007).

In this framework, orbital decay and stellar spin-up only occur if the stellar rotation rate is lower than the planetary orbital revolution rate, $n$. In other words, the orbit decays if the stellar rotation period is larger than the orbital period of three days and even less for companions on very tight orbits, as considered in this work. If the stellar rotation rate and revolution rate are equal, that is, synchronised, tidal friction and, thus, orbital decay stops in the absence of magnetic braking.

Given the tidal evolution of the semi-major axis (Eq. (1)), we can derive the equations for the rate of change of the stellar rotation rate, $\Omega_{\star}$, via angular momentum transport of the orbit towards the host star. The dominant fraction of the planetary angular momentum is stored in the planetary orbit. Therefore, changes in the planetary spin can be neglected (e.g Carone 2012; Murray \& Dermott 1999). Thus, the total angular momentum $L$ of the star - planet system mainly consists of the component stored in the stellar spin and in the companion orbit (cf. Murray \& Dermott 1999):

$L=I_{\star} \Omega_{\star}+\frac{M_{\star} M_{\mathrm{pl}}}{M_{\star}+M_{\mathrm{pl}}} a^{2} n$,

where $I_{\star}$ is the moment of inertia of the star defined as $I=$ $k^{2} M_{\star} R_{\star}^{2}$ and $n$ is, again, the orbital revolution rate.

When Eqs. (1) and (5) are combined ${ }^{4}$, the tidal spin-up can approximately be described by:

$$
\begin{aligned}
\left(\frac{d \Omega_{\star}}{\mathrm{d} t}\right)_{\text {tide }} & =-\frac{M_{\mathrm{pl}} M_{\star}}{2 I_{\star}\left(M_{\mathrm{pl}}+M_{\star}\right)} n a \dot{a} \\
& =-\frac{1}{2 k^{2}} \frac{P_{\mathrm{pl}}}{2 \tau^{3}}\left(\frac{M_{\mathrm{pl}}}{M_{\star}}\right)^{2}\left(\frac{R_{\star}}{a}\right)^{6} \frac{M_{\mathrm{env}}}{M_{\star}},
\end{aligned}
$$

The $k^{2}$-value ranges from 0.03 for fully radiative stars to 0.2 for fully convective stars (Nelemans et al. 2001). For all calculations where we focus on MS stars with a thick enough convective outer envelope that promotes efficient tidal interaction, $k^{2}$ is set to the solar value of 0.074 , which was calculated based on moment of inertia calculated from the mass-radius distribution based on the helioseismology data of Dziembowski et al. (1994). We confirmed in our stellar models that $k^{2}$ differs at most by $30 \%$ from this value. See also Bouvier et al. (1997) and their Fig. 1; these authors also focus on MS stars with masses of 0.8 and $1 M_{\odot}$.

The tidal model adopted in this work is valid for stars with a thick convective envelope that allows for an efficient tidal dissipation of energy. The efficiency limit of stellar tides can also be

4 Here, we assume that $\dot{L}=0$ and $\dot{I} \approx 0$ during the MS. Further, it can be shown that $\frac{d}{\mathrm{~d} t}\left(a^{2} n\right)=\frac{1}{2} a \dot{a} n$, using Kepler's law $n=$ $a^{-3 / 2} \sqrt{G\left(M_{\mathrm{Pl}}+M_{\star}\right)}$. 
inferred from their effects on the obliquity in a planetary system, that is, the angle between a planetary orbital plane and the stellar rotation axis. The obliquity can be measured via the RossiterMcLaughlin effect during the transit of close-in giant planets (or hot Jupiters). See Queloz et al. (2010) for the first such measurement for an exoplanet. In addition, Winn et al. (2010) and Albrecht et al. (2012) pointed out that based on the measured obliquities of Jupiters, it appears that, originally, shortly after formation, the obliquities of hot Jupiters can be very large. These large obliquities are then damped afterwards to small values via tides, provided the host star is cool enough to have a thick convective envelope. The limit of tidal efficiency was determined to be at $M_{\text {env }} / M_{\star} \approx 0.005$ (Winn et al. 2010, Fig. 2) and for smaller values, the tides are negligible. For stars of solar metallicity and higher this corresponds to strong tides and small obliquities for $T_{\text {eff }}<6250 \mathrm{~K}$, which is in accordance with observations of the stellar spin-orbital plane angle for systems with hot Jupiters via the Rossiter-McLaughlin effect (Winn et al. 2010). We have taken the mass and temperature thresholds into consideration and for metal-poor stars, which are hotter, exclude those cases for which $M_{\mathrm{env}} / M_{\star} \approx 0.005$.

\subsection{Loss of angular momentum by magnetised stellar winds}

To coherently study stellar evolution over the MS, we also take into account the evolution of stellar rotation rate caused by the loss of angular momentum by magnetised winds. In the following, we use the model of Bouvier et al. (1997):

$\frac{1}{\Omega_{\star}}\left(\frac{d \Omega_{\star}}{\mathrm{d} t}\right)_{\text {wind }}=\frac{1}{J}\left(\frac{d J}{\mathrm{~d} t}\right)-\frac{1}{I} \frac{d I}{\mathrm{~d} t}$,

where $J$ is the angular momentum of the star $J=I \Omega_{\star}$, assuming solid body rotation. Stellar evolution models are used to calculate the evolution of $d I / \mathrm{d} t$ with time. The change of angular momentum of a star is, furthermore, described by:

$$
\begin{aligned}
& \frac{d J}{\mathrm{~d} t}=-K \Omega_{\star}^{3}\left(\frac{R_{\star}}{R_{\odot}}\right)^{1 / 2}\left(\frac{M_{\star}}{M_{\odot}}\right)^{-1 / 2} \quad \text { for } \Omega_{\star} \leq \omega_{\text {sat }}, \\
& \frac{d J}{\mathrm{~d} t}=-K \omega_{\mathrm{sat}}^{2} \Omega_{\star}\left(\frac{R_{\star}}{R_{\odot}}\right)^{1 / 2}\left(\frac{M_{\star}}{M_{\odot}}\right)^{-1 / 2} \text { for } \Omega_{\star}>\omega_{\text {sat }},
\end{aligned}
$$

where $K$ is a phenomenological scaling factor that Bouvier et al. (1997) introduced to connect observational rotation rates from stellar clusters of different ages to the observed small rotation rates of MS stars like the Sun. Here, $\omega_{\text {sat }}$ is the rotation rate, for which the magnetic field is assumed to saturate. With faster rotation, magnetic activity and, thus, magnetic braking no longer increases strongly, following the dynamo relation at the basis of this formulation. Consequently, also the stellar angular momentum loss saturates and no longer increases with faster rotation. In addition, $\omega_{\text {sat }}$ is typically constrained by observations to explain the spread in rotational velocities in zero age main sequence (ZAMS) clusters. It was scaled by Bouvier et al. (1997) to be $14 \Omega_{\odot}=\Omega_{\text {sat }}$ for $1 M_{\odot}$ stars. Although the scaling varies with mass, we adopt the aforementioned value to make the computations more readily comparable to other studies in the field. Amard \& Matt (2020) adopt a single value as well, $\Omega_{\text {sat }}=11 \Omega_{\odot}$, for masses between $0.8-1.3 M_{\odot}$ stars. The evolution starts with $P_{\text {ini }}$. The values for $K, \omega_{\text {sat }}$, and $P_{\text {ini }}$ for different masses are listed in Table 1. Figure 3 shows the application of the Bouvier model and that by the time a star reaches solar age, regardless of the initial conditions, the magnetic braking has sufficiently slowed down the star to a solar value.
Table 1. Parameters for the stellar rotation evolution.

\begin{tabular}{lc}
\hline \hline Parameter & Value \\
\hline Disk locking time $\left(\tau_{\text {disc }}\right)$ & $1 \mathrm{Myr}$ \\
Saturation velocity $\left(\omega_{\text {sat }}\right)$ & $14 \Omega_{\odot}$ \\
Scaling factor $(\mathrm{K})$ & $2.7 \times 10^{47}$ \\
Initial rotation period $\left(P_{\text {ini }}\right)$ & 7.8 days \\
Initial angular velocity $\left(\Omega_{0}\right)$ & $9.32 \times 10^{-6} \mathrm{~s}^{-1}$ \\
Solar angular velocity $\left(\Omega_{\odot}\right)$ & $2.9 \times 10^{-6} \mathrm{~s}^{-1}$ \\
\hline
\end{tabular}

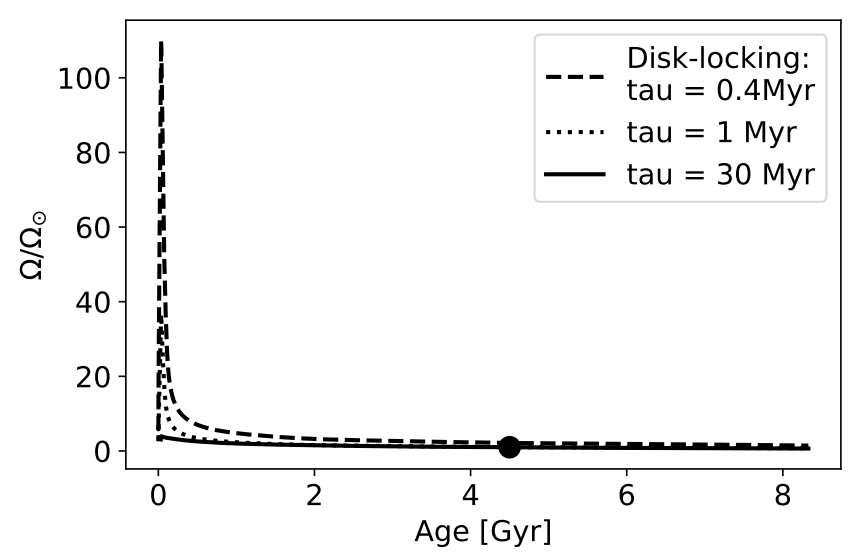

Fig. 3. Example simulation of rotation velocity according to the Bouvier et al. (1997) for a $1 M_{\odot}$ model. The black dot indicating the solar value at 4.5 Gyr. The input parameters are presented in Table 1. Shown are three different curves for different disk locking time, i.e. the time it takes the star to decouple from its surrounding protoplanetary disk.

During the tidal migration, the total effect of magnetic braking and tidal interaction is calculated using:

$$
\frac{d \Omega_{\star}}{\mathrm{d} t}=\left(\frac{d \Omega_{\star}}{\mathrm{d} t}\right)_{\text {wind }}-\frac{1}{2 k} \frac{P_{\mathrm{pl}}}{2 \tau^{3}}\left(\frac{M_{\mathrm{pl}}}{M_{\star}}\right)^{2}\left(\frac{R_{\star}}{a}\right)^{6} \frac{M_{\mathrm{env}}}{M_{\star}} .
$$

Although we assume that tidal dissipation takes place mainly in the outer convective envelope of the star, the angular momentum transfer from the planetary orbit spins up the star as a whole, assuming solid body rotation.

We note that there have been recent improvements on the Bouvier model to improve stellar evolution during the pre-mainsequence more coherently (Gallet \& Bouvier 2013, 2015). Since we focus on the effect of tidal migration $\left(\tau_{\text {tide }} \geq 1 \mathrm{Gyrs}\right)$, which affects the star only when it is already on the MS, the simpler Bouvier model is sufficient for this work.

We note also that the Bouvier model that we use here is based on large parts on the model of Kawaler (1988). The latter model is used in recent work by Amard \& Matt (2020) to represent spin-down rates due to "nominal" magnetic braking compared to reduced magnetic braking for massive, metalpoor stars $\left(M_{\star} \geq 1 M_{\odot}\right)$ over timescales of billion of years. Amard \& Matt (2020) also stress the importance of stellar structure in their work, which indicates that the absence of a thick convective envelope is responsible for reduced magnetic braking.

Conversely, in this work, we focus on less massive $\left(M_{\star} \leq\right.$ $\left.1 M_{\odot}\right)$, metal-poor stars, because for efficient tidal interactions a thick enough convective envelope is required. A thick convective envelope promotes at the same time efficient magnetic braking 
A. Oetjens et al.: The influence of planetary engulfment on stellar rotation in metal-poor main-sequence stars

as we simulate in this work. We stress again that we always check with stellar modelling that a convective layer thicker than $0.005 M_{\star}$ is present when applying our star-planet interaction models.

\subsection{Stellar spin-up due to tidal friction}

As mentioned previously, we consider tidal migration of substellar mass companions on tight orbits (0.03 AU and shorter or for orbital periods shorter than three days). At the same time, the star loses its angular momentum via magnetic braking.

The choice of initial conditions is motivated by the following considerations. A Sun-like star with a mass of $0.8-1 M_{\odot}$ and an age of $4.5 \mathrm{Gyr}$ typically rotates with the velocity of 25.1 days $\left(\Omega=\Omega_{\odot}\right)$. The pre-main-sequence phase of a star is much shorter than the characteristic timescales of tidal migration $\left(\tau_{\text {tide }} \geq 1 \mathrm{Gyr}\right)$. Therefore, we assume for the tidal migration scenario that the planetary orbital period $P_{\mathrm{pl}}$ is initially significantly smaller than the stellar (surface) rotation period $P_{\star}$ at the time when the sub-stellar companion starts to migrate inwards. In such a situation, the crest of the tidal bulge on the star, induced by the companion, always lags behind with respect to the line connecting the centre of masses of the companion, the star, and the tidal bulge. Therefore, the tidal torque acts in the direction of stellar rotation leading to the spin-up of the star. The orbit of the companion shrinks due to the conservation of the total angular momentum (Fig. 2).

The decay of the planetary orbit depends on two quantities. One is the total angular momentum $L$ in the system. The other is the relationship between the orbital revolution rate $n$ of the planet and the rotation rate of the star $\Omega_{\star}$ (Table 2). In this work, we consider two cases: the tidal inwards migration $\left(\Omega_{\star}<n\right)$ and the double synchronicity $\left(\Omega_{\star}=n\right)$. Both scenarios are shown in Fig. 4 and briefly described below. If $\Omega_{\star}>n$, that is, the companion is beyond a critical distance, the tidal migration is reversed and the companion migrates outward. In this configuration, angular momentum is transferred from the host star to the companion and, in fact, a decrease in the stellar rotation rate occurs. A similar phenomenon occurs in the Earth-Moon system, where tidal friction acts to slow down the Earth's rotation as the Moon migrates away from the Earth.

\subsubsection{Tidal inwards migration}

In this scenario, tidal friction causes the companion (a planet or a brown dwarf) to migrate inwards and to gradually spin up the star. We run the simulation up until the point when the star has approached the MS turnoff point or when the planet has reached the Roche limit, the distance at which a planet will disintegrate due to the strong differential of tidal forces inside the body exceeding the planetary gravitational self-attraction which is defined as:

$d_{\text {Roche }}=R_{\star}\left(2 \frac{\rho_{\star}}{\rho_{\mathrm{pl}}}\right)^{1 / 3}$.

The Roche limit varies between 1.44 and $2.44 R_{\star}$ (Murray \& Dermott 1999). We adopt a conservative assumption that the Roche limit is located at $1.44 R_{\star}$ and that angular momentum is no longer transported towards the star upon the disruption of the planet. That is, we conservatively assume that upon disruption, the remaining angular momentum of the planet is removed from the system. If some angular momentum is
Table 2. Notations and variables adopted in this work.

\begin{tabular}{lcc}
\hline \hline Parameter & Notation & Unit \\
\hline Star & & \\
Rotation period & $P_{\star}$ & days \\
Angular frequency of rotation & $\Omega_{\star}=\frac{2 \pi}{P_{\star}}$ & $\mathrm{rad} \mathrm{s}^{-1}$ \\
Rotation velocity & $v_{\mathrm{rot}}=\Omega_{\star} R$ & $\mathrm{~km} \mathrm{~s}^{-1}$ \\
Mass & $M_{\star}$ & $\mathrm{g}$ \\
Mass of CE ${ }^{(a)}$ & $M_{\mathrm{env}}$ & $\mathrm{g}$ \\
Radius at the bottom of CE ${ }^{(a)}$ & $R_{\mathrm{env}}$ & $\mathrm{cm}$ \\
\hline Planet & & \\
Orbital period & $P_{\mathrm{pl}}$ & $\mathrm{days}$ \\
Semi-major axis of the orbit & $a$ & $\mathrm{AU}$ \\
Initial semi-major axis & $a_{0}$ & $\mathrm{AU}$ \\
Planetary revolution rate & $n$ & $1 \mathrm{~s}^{-1}$ \\
Mass & $M_{\mathrm{pl}}$ & $\mathrm{g}$ \\
\hline
\end{tabular}

Notes. ${ }^{(a)}$ CE denotes the convective envelope.

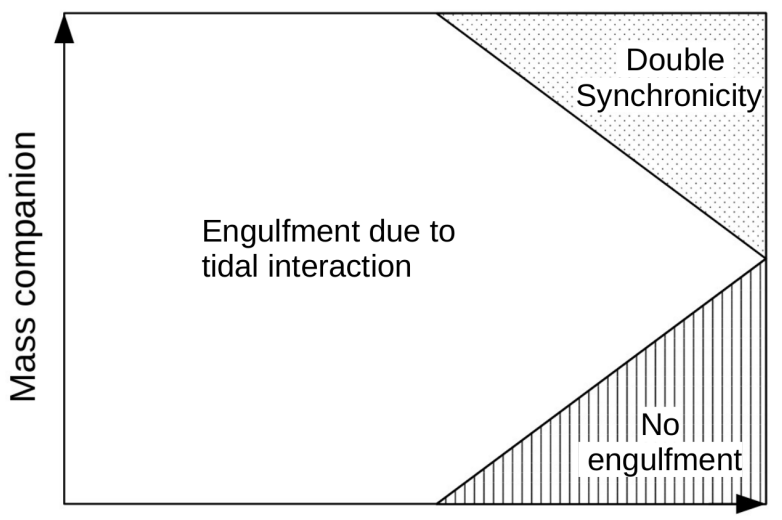

Initial semi-major axis

Fig. 4. Outcomes of tidal interaction between a star and its companion in our model: engulfment (white area), double synchronicity (upper right corner), and no significant interaction up until the MS turnoff point (lower right corner).

still transported to the star during disruption, the star would be spun up even faster. Therefore, the final rotation rate of a star obtained in our simulations is a lower limit ${ }^{5}$.

Furthermore, the timescale of planetary migration is one of the most important quantities in our model because it defines two possible outcomes of the dynamical interaction, which we refer to as fast and the slow migration; these are illustrated in Fig. 5. If the timescale of tidal migration is shorter than the MS lifetime of a star, $\tau_{\text {tide }} \leq \tau_{\text {life }}$, the sub-stellar companion reaches the Roche limit within the MS lifetime of a star and is destroyed (Fig. 5, top panel). On the other hand, if $\tau_{\text {tide }}>\tau_{\text {life }}$, the substellar companion does not reach the Roche limit within the MS lifetime of the star. In both cases, the rotation velocity of the host star can be significantly increased (Fig. 5, bottom panel). Previous works in this field (see e.g. Carone \& Pätzold 2007; Bolmont et al. 2017, namely, Figs. 2 and 4; Fig. 6c) explore the effects of tidal interaction and magnetic braking in the context of planetary engulfment. The amount of angular momentum stored in the planetary orbit is much larger compared to

\footnotetext{
5 As a reminder, higher rotation rates mean faster rotation and shorter
} rotation periods. 

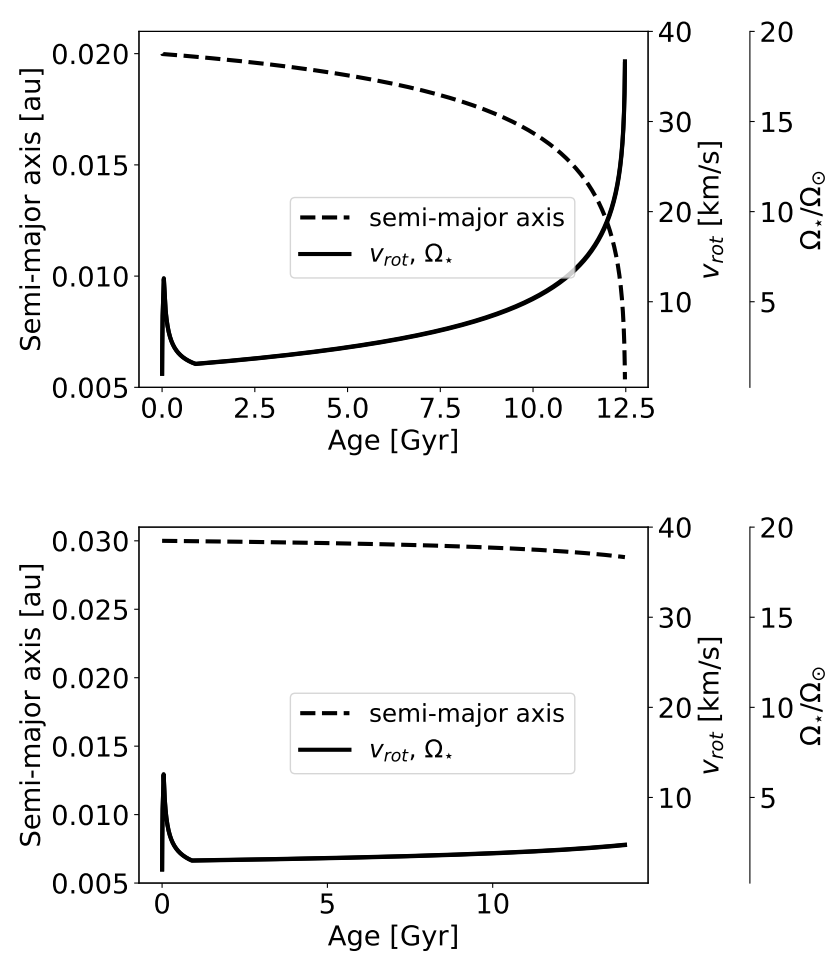

Fig. 5. Top: evolution of the semi-major axis (dashed line) and the rotation velocity (solid line) of a $0.8 M_{\odot}$ star with $[\mathrm{Fe} / \mathrm{H}]=-0.4$ and a $10 M_{\text {Jup }}$ companion at a distance of $a_{0}=0.02$ AU. Bottom: same conditions for the same stellar model with a $10 M_{\text {Jup }}$ companion at $a_{0}=0.03 \mathrm{AU}$. No engulfment occurs during the MS lifetime.

the stellar rotation, which reduces the efficiency of the magnetic braking mechanism. At the same time, magnetic braking tends to saturate for fast rotating stars (here $14 \Omega_{\odot}$ ) and thus it cannot compensate for the continuously increasing angular momentum transfer as the companion moves further in. The details of how much the star can be spun up, however, depend on when planetary engulfment (and thus angular momentum transport) is completed. For instance, Carone \& Pätzold (2007) assumed that planetary engulfment stops at a Roche limit of $2.4 R_{\star}$ for a $1.5 M_{\text {Jup }}$ planet, while Bolmont et al. (2017) find that planetary engulfment stops at $0.01 \mathrm{AU}$ for a $1 M_{\text {Jup }}$ planet. In this work, we assume a Roche limit of $1.44 R_{\star}$.

\subsubsection{Double synchronicity}

If $\Omega_{\star}=n$, that is, when the stellar rotation rate is equal to the planetary revolution rate, there is no tidal friction and, thus, no torque that can spin up the star or lead to the orbital decay. Hut (1980) showed that when the total angular momentum of a binary system exceeds a critical value, $L_{\text {crit }}$, the system can end up in a double synchronous rotation and thus, to first order, it is in a stable configuration, preventing further migration inwards. We stop the calculations once $\Omega_{\star}=n$, that is, when the double synchronicity is reached for $L>L_{\text {crit }}$. It is, in principle, possible that the angular momentum of the system can drop below $L_{\text {crit }}$ later in the evolution of the star (Carone 2012; Guillot et al. 2014) because of further angular momentum loss due to magnetic braking. The set of tidal equations that we use here are not suitable to simulate the further evolution of a double synchronous state where: a) the tidal bulges raised by the planet no longer move with respect to the surface of the star; and b) the dynamical tide, and not the equilibrium tide, becomes important (Ogilvie \& Lin 2007). Thus, a separate treatment and different set of tidal equations is required (Carone 2012; Guillot et al. 2014). We do not consider this scenario in this work, but we postpone its treatment to the next paper in the series. We note, however, that previous works suggest that such systems may only be pseudo-stable and will eventually destabilize, if $L<L_{\text {crit }}$ is reached within the life time of the system (Hut 1981; Carone 2012; Guillot et al. 2014). In this case, the planetary companion will start to migrate inwards again (Damiani \& Díaz 2016; Hodžić et al. 2018).

\subsection{Tidal interaction during the PMS}

While we focus on the tidal interaction during the MS, it is worthwhile discussing the impact of planet migration during the premain sequence (PMS) and how this may affect the occurrence rate of close-in massive planets and brown dwarfs on the MS.

During the PMS, the equilibrium tide plays a negligible role and, thus, the dominant cause for tidal interaction is the dynamical tide. After reaching the MS, the dynamical tides are surpassed in strength by the equilibrium tides, which justifies our decision to neglect dynamical tides in this work. For an in-depth discussion and derivation the reader is referred to Rao et al. (2018), Bolmont et al. (2017), Heller (2019). They further suggested that dynamical tides during the PMS are so efficient that many close-in planets (located within $0.04 \mathrm{AU}$ ) around solarmass stars could either be tidally engulfed during the PMS or migrate outwards. In both cases, no planetary engulfment occurs during the MS. In the first case, the planet no longer exists while the star is on the MS and in the second case, the planet is too far away for the weaker equilibrium tides to achieve planetary engulfment during the MS.

As noted by the authors, the exact tidal migration scenario depends very strongly on the initial stellar rotation period. It has been consistently shown that mainly planets orbiting stars with initial stellar rotation periods of two days and shorter are prone to either becoming engulfed within the PMS or to migrating too far outwards for later planetary engulfment during the MS. Furthermore, dynamical tides in metal-poor stars $([\mathrm{Fe} / \mathrm{H}]=-0.5)$ are much less efficient. Thus, even planets on short orbits - within $0.04 \mathrm{AU}$ - could survive while the star is in the PMS evolutionary stage, particularly when they orbit a host star with an initial stellar rotation period of six days and longer (Bolmont et al. 2017, Fig. 7). In addition, observed stellar rotation in young ( $\lesssim 5$ Myrs) stellar clusters show that a substantial fraction of Sunlike stars appear to have rotation periods of six days and longer (Bouvier et al. 1997; Irwin et al. 2008). Also, while the findings of Rao et al. (2018) and Bolmont et al. (2017) predict the engulfment of companions before reaching the MS for certain conditions, there is evidence that these systems do exist. WASP-19 is a solar-like MS-star with a close-in (0.017 AU) $1.2 M_{\text {Jup }}$ companion (Hebb et al. 2010).

In conclusion, tidal interaction between the star in the PMS evolutionary stage and its planetary companion can have a significant effect on individual planetary systems. However, in this work, we want to stress that stellar spin-up during the MS due to tidal migration on the MS can occur and could be observable due to excess of rotational velocity in the host star. The observation of abnormally fast (exceeding model predictions) rotators on the MS would then justify more detailed evolution studies from the PMS to the MS. 


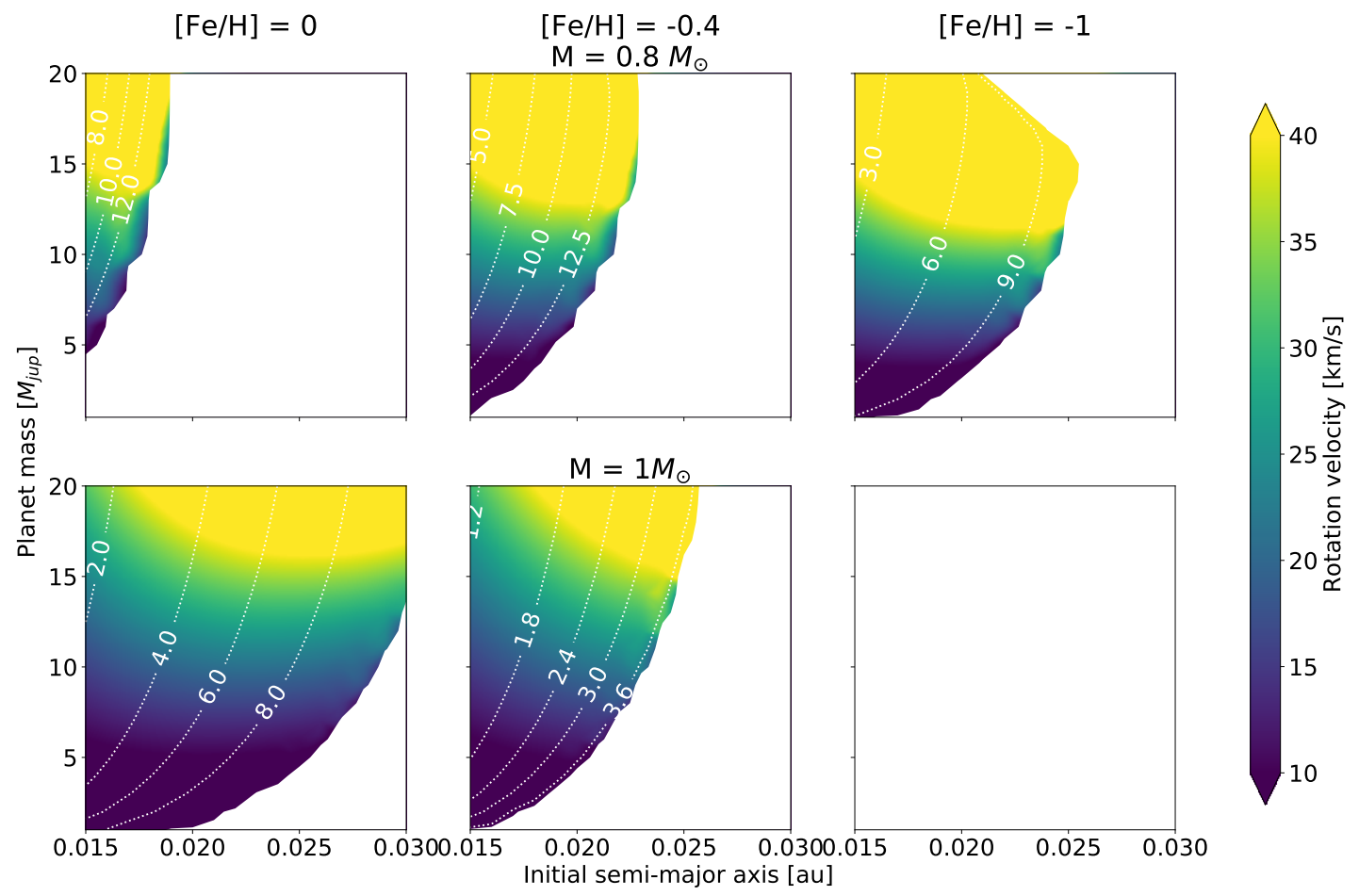

Fig. 6. Tidal maps: computational results. We colour-code the stellar spin-up depending on the planetary mass and initial semi-major axis. The white lines give the time of engulfment in Gyr. The white areas correspond to regions where no engulfment occurs.

\section{Results}

Here we present the results of running our tidal evolution model for several combinations of input parameters, which include the mass and metallicity of a star, as well as the mass and initial semi-major axis of the companion (a massive planet).

Figure 6 shows the rotation rate of the star after engulfment in the $a_{0}-M_{\mathrm{pl}}$ plane, that is, the initial semi-major axis of the planet ( $x$-axis) and its mass ( $y$-axis). We show six stellar models, each one for different metallicities $([\mathrm{Fe} / \mathrm{H}]=0,-0.4,-1)$ and masses $\left(M=0.8,1 M_{\odot}\right)$. The age of the star at the time of engulfment is also indicated. The simulation stops either when the companion reaches the Roche zone, which causes its tidal disruption, when the star reaches the MS turn off before planetary engulfment has occurred, or when the mass of the convective envelope drops below the critical limit. The latter two situations are represented by blank spaces in the upper and lower right corner of the graph. Systems in double synchronicity (Sect. 2.3) are not explored and are represented as blank spaces in the upper right corner of the tidal maps. Clearly, $a_{0}$ and $M_{\mathrm{pl}}$ are two important parameters that influence the outcome of the simulation: more massive planets and planets on larger orbits lead to a more efficient spin-up of a star (see also Fig. 5) because most of the angular momentum is stored in the orbit of the planet around the star. On the other hand, the time to engulfment increases with increasing the initial semi-major axis of the planet.

Comparing the results for the 0.8 and $1 M_{\odot}$ models, we find that engulfment is much more efficient - and, thus, more likely for more massive stars: the $1 M_{\odot}$ star (Fig. 6, bottom row) experiences a significant spin-up within the entire parameter space explored in this work. This, however, is only true as long as a convective envelope thick enough for efficient tidal interaction $\left(M_{\text {env }} / M_{\star} \geq 0.005\right)$ is present. Therefore, at $[\mathrm{Fe} / \mathrm{H}]=-1$, the $1 M_{\odot}$ is excluded as the mass of the convective envelope is already below this limit. Even low-mass companions, $M_{\mathrm{pl}}<$
$10 M_{\mathrm{Jup}}$, on orbits from 0.015 to $0.03 \mathrm{AU}$ are capable of transferring enough angular momentum to spin up the star by up to $20 \mathrm{kms}^{-1}$ from the initial rotation rate of $2 \mathrm{kms}^{-1}$. Also, the engulfment timescales are much shorter for more massive stars. For the $1 M_{\odot}$ star at $[\mathrm{Fe} / \mathrm{H}]=-0.4$, the short-period massive planet will be engulfed within 1-3 Gyr, whereas for the $0.8 M_{\odot}$ star the process will take up to more than 12 Gyr. On the other hand, comparing the amplitudes of spin-up (i.e. the resulting rotation velocity of a star), we find that the impact on the lessmassive star is larger. For example, the engulfment of a planet with $M_{\mathrm{pl}} \approx 15 M_{\mathrm{Jup}}$ and the initial semi-major axis of $0.015 \mathrm{AU}$ increases the rotation rate of the $0.8 M_{\odot}$ star by a factor of 40 . In contrast, the more massive star with a similar companion will only spin up by a factor of 25 , although the engulfment occurs much faster. This is because, efficient tidal interaction requires a thick convective envelope to dissipate tidal energy within the star. Adding to that is the effect that smaller mass stars have lower moments of inertia because of smaller mass and smaller radii on the MS.

In addition, the metallicity of the star has a significant influence on the outcome of the star-planet interaction. This is best seen by comparing our results for $[\mathrm{Fe} / \mathrm{H}]=0$ and -1 model (Fig. 6, top panel). A solar metallicity $0.8 M_{\odot}$ star requires more than $12 \mathrm{Gyr}$ to engulf a $10 \mathrm{M}_{\mathrm{Jup}}$ companion at an initial distance of 0.017 AU. This results in a rotation velocity of $\sim 30 \mathrm{kms}^{-1}$. This process is three times faster for a star of the same mass, but much lower metallicity, $[\mathrm{Fe} / \mathrm{H}]=-1$ : The companion is engulfed in less than 5 Gyr. The chemical composition of a star is, therefore, a very important parameter, along with the mass of a star, which has a strong influence on the evolution of the orbit of the planet and the likelihood of engulfment.

The behaviour of spin-up rates with stellar mass and metallicity can be explained by the differences in stellar structure. For a given mass, more metal-poor stars are larger, have smaller convective envelopes, and evolve faster on the MS. Calculations 
with our model suggest that such a physical configuration that is, a smaller convective envelope and larger stellar radius favours a faster engulfment. However, it should be stressed that the convective envelope must be thick enough to efficiently dissipate tidal energy. Also, beyond some critical combination of mass and metallicity (here $1 M_{\odot}$ and $[\mathrm{Fe} / \mathrm{H}]=-1$ ), planetary engulfment is no longer possible, as the mass of the convective envelope is too low for efficient tidal dissipation (Winn et al. 2010).

\section{Discussion}

Our results suggest that tidal interaction between a star and its companion - a massive planet or a brown dwarf - may lead to a significant spin-up of the host star. This can be the consequence of the engulfment of a companion, but it is also true in the case of the double synchronous rotation - a stable configuration that prevents the engulfment and disruption of a companion. The timescales of interaction can vary significantly, from a few hundred Myr up to $\gtrsim 12 \mathrm{Gyr}$, and they depend on several parameters, among them the initial semi-major axis of the planet, the mass of the planet and its host star, and stellar metallicity.

The main prediction of our model is that at low metallicities, a larger fraction of planets that dwell in the conditions considered in this study are engulfed by their host star, resulting in abnormal rotation velocities. Indeed, lower-mass stars have significantly longer MS lifetimes, and lower metallicity implies a smaller convective envelope, shorter convective turnover time, and a larger stellar radius, thus favouring a faster engulfment. While the impact of an increasing stellar radius is obvious from the evolution of the semi-major axis, the rate of orbital decay is also a function of the mass of the convective envelope and the convective turnover time $(\tau)$. Since the numerical factor is $f \sim 1 / \tau^{2}$ in all our scenarios, the rate of orbital decay follows as:

$\left(\frac{\dot{a}}{a}\right)_{\text {tidal }} \sim\left(\frac{M_{\text {env }}}{\tau^{3}}\right)$

With decreasing metallicity, we have $M_{\text {env }} / \tau^{3}>1$, where the orbital decay rate increases and thus leads to faster planetary engulfment. This was also shown by Amard \& Matt (2020), who illustrate changes in the convective turnover time for metal-poor $([\mathrm{Fe} / \mathrm{H}]=-1)$ stars.

The prediction of abnormally fast rotation velocities that surpass any model predictions, particularly for stars with sub-solar metallicity, is a strong observational signature. It could, in principle, be detectable in samples of stars with accurate measurements of ages, metallicities, and rotation periods. It depends, however, on the occurrence rate of close giant planets across stellar metallicities. We have, therefore, analysed multiple catalogues in the literature in the attempt to find stars with reliable estimates of metallicity and short rotation periods (Huber et al. 2014; McQuillan et al. 2014). However, it has unfortunately turned out the current sample of statistics is too small and does not allow us to probe the critical metallicity range, $[\mathrm{Fe} / \mathrm{H}] \lesssim-1$. This is most likely because metallicity estimates of fast rotators are difficult, as the spectral lines are significantly broadened and can barely be distinguished from the continuum at $[\mathrm{Fe} / \mathrm{H}] \lesssim-1$, especially if the signal-to-noise ratio $(\mathrm{S} / \mathrm{N})$ of the observed data is not very high. This implies that targeted surveys must be performed to find fast-rotators, which are candidates for being old and metal-poor.

The lack of a significant population of observed stars with abnormal rotation does not necessarily stand as evidence for the failure of our model. Complementary insights on the feasibility of this scenario can be gained from the observations of planets in discs. There are several planetary companions and planetesimals on short orbital periods that are known to be currently spiralling inwards toward their host stars. Vanderburg et al. (2015) discovered an evaporating, small, gas planet around the white dwarf, WD 1145+017. Gänsicke et al. (2019) reported a disintegrating dust ring around the white dwarf, WD J091405.30+191412.25. WASP-19b (Espinoza et al. 2019) and NGTS-10b (McCormac et al. 2020) are hot Jupiters on ultra-short orbital periods ( $<1$ days); they may spiral towards their host star within a few tens of Myr, leading to a significant spin-up of the host star.

Another interesting consequence of our planetary engulfment scenario is that it could influence the surface chemical composition of the star. Indeed, peculiar abundances were reported for different types of stars in single and binary systems, for example, solar twins, FG-type MS binaries, and white dwarfs (e.g. Meléndez et al. 2012; Spina et al. 2015; Teske et al. 2016; Petrovich \& Muñoz 2017; Nissen et al. 2017; Veras \& Fuller 2019). A recent study by Oh et al. (2018) provided interesting observational evidence for the accretion of a massive rocky planet $\left(\sim 15 M_{\text {earth }}\right)$ in a $\sim 4$ Gyr old binary system. Detailed theoretical predictions for this process do not yet exist, but it has already been shown that even the accretion of dust and gas from a proto-planetary disc may lead to a significant alteration of the surface abundances of stars (Kunitomo et al. 2018; Hoppe et al. 2020). We leave the analysis of the potential influence of the engulfment on the stellar chemical composition to the next paper in the series.

Finally, we would like to briefly discuss our work in the context of another scenario that was recently proposed by Amard \& Matt (2020). They suggest that the phenomenon of magnetic braking is not efficient enough to slow down the rotation rate for metal-poor systems. The modified equations introduced by Amard \& Matt (2020) are, however, conceptually very similar to the equations that we used to simulate spin-down (Sect. 2.2). Thus, it will be easy to adapt our model framework to simulate both effects in conjunction and to produce an even clearer picture if it can be shown that one of the mechanisms (tidal engulfment or inefficient magnetic braking) or both may be responsible for rapidly-rotating low-metallicity stars.

\section{Conclusions}

In this work, we study the influence of tidal interaction between a star and its companion on the rotation velocity of the star. This dynamical interaction is followed using a detailed numerical framework that combines tidal friction, magnetic braking, planet migration, and detailed stellar structure models from the GARSTEC grid. We focus on close-in massive planetary companions with masses from 1 to $20 M_{\text {Jup }}$ orbiting low-mass, 0.8 and $1 M_{\odot}$, MS stars within a broad metallicity range from $[\mathrm{Fe} / \mathrm{H}]=-1$ to solar.

We find that planetary engulfment of massive planetary companions on tight orbits around their host stars can significantly accelerate stellar rotation on timescales of several billion years. Companions with masses between 5 and $20 M_{\text {Jup }}$ (the latter is the maximum mass probed in our simulation) and initial semi-major axis $<0.03 \mathrm{AU}$ lead to a spin-up of the star up to $40 \mathrm{kms}^{-1}$.

All the parameters, namely, the initial semi-major axis of the planet and its mass, along with the mass and metallicity of the star, are relevant in determining the final rotation rate of the star. In most cases, the model predicts that the companion is 
engulfed and the timescales of the process are shorter for more massive host stars. Tidal migration timescales are also shorter for metal-poor stars. This is because low-metallicity systems have a larger radius and a less massive convective envelope, which facilitates efficient tidal interaction and favours an engulfment of the companion. This, however, is only true as long as the convective envelope is above the limit of $0.005 M_{\odot}$. Due to the higher mass and, therefore, a higher moment of inertia, for a $1 M_{\odot}$ star compared to its low-mass $\left(0.8 M_{\odot}\right)$ counterpart, the less massive star reaches higher rotation velocities. We also find that the fastest planetary engulfment occurs for the model with $1 M_{\odot}$ and $[\mathrm{Fe} / \mathrm{H}]=-0.4$. The largest stellar rotational velocities for a given planetary mass and initial semi-major axis are reached for $0.8 M_{\odot}$ and $[\mathrm{Fe} / \mathrm{H}]=-1$.

In certain ranges of the investigated parameter space, however, no engulfment occurs and the planet forms a stable synchronized system with its host stars. This outcome is seen, for example, for the $0.8 M_{\odot}$ and $[\mathrm{Fe} / \mathrm{H}]=-1$ model. Also in this case, the rotation of the host star is significantly increased.

Our model predicts that low-mass MS stars, owing to the tidal interaction with their close-in companion, can experience a significant spin-up in their surface rotation. Furthermore, stars with abnormal rotation velocities should be more common at low-metallicity, if occurrence rates for close-in massive planets is similar to that of more metal-rich stars, as lower $[\mathrm{Fe} / \mathrm{H}]$ favours faster planet engulfment. Current observational samples are too small and incomplete and thus, they do not allow us to test our model. However, future studies, targeting rapidly-rotating stars with low metallicity, can provide enough statistics to test the main observable signature of our scenario. Also, the system in a stable synchronized state should, in principle, be detectable via multi-epoch radial velocity measurements.

In any case, it is clear that for the study of tidal interactions of close-in planets (in our model $a_{0} \leq 0.03 \mathrm{AU}$ ), the evolution of stellar rotation is an important parameter. This is true for the PMS (Rao et al. 2018; Bolmont et al. 2017) and for the MS stars. Furthermore, the investigation of abnormal fast rotation in metalpoor MS stars is immensely valuable to the investigatation of several fundamental processes: in probing the limits of applicability of magnetic braking to explain the stellar rotation evolution of MS stars and investigating the formation of massive planets and low-mass brown dwarfs in the close vicinity of metal-poor, solar-like stars.

Acknowledgements. $\quad$ L. C. acknowledges support by the DFG Grant CA 1795/3. We acknowledge support by the Collaborative Research centre SFB 881 (projects A5, A10), Heidelberg University, of the Deutsche Forschungsgemeinschaft (DFG, German Research Foundation). A. S. is partially supported by MICINN grants ESP2017-82674-R and PID2019-108709GB-I00, and Grant 2017-SGR1131 from Generalitat of Catalunya. We thank the anonymous referee for the insightful and constructive comments.

\section{References}

Albrecht, S., Winn, J. N., Johnson, J. A., et al. 2012, ApJ, 757, 18 Amard, L., \& Matt, S. P. 2020, ApJ, 889, 108

Angus, R., Aigrain, S., Foreman-Mackey, D., \& McQuillan, A. 2015, MNRAS, 450,1787

Angus, R., Morton, T. D., Foreman-Mackey, D., et al. 2019, AJ, 158, 173

Barnes, S. A. 2003, ApJ, 586, 464

Barnes, S. A. 2007, ApJ, 669, 1167

Barnes, R. 2015, Int. J. Astrobiol., 14, 321

Beck, P. G., Mathis, S., Gallet, F., et al. 2018, MNRAS, 479, L123

Beck, P. G., Montalban, J., Kallinger, T., et al. 2012, Nature, 481, 55

Bolmont, E., Gallet, F., Mathis, S., et al. 2017, A\&A, 604, A113

Bouvier, J., Forestini, M., \& Allain, S. 1997, A\&A, 326, 1023

Buchhave, L. A., Latham, D. W., Johansen, A., et al. 2012, Nature, 486, 375
Carone, L. 2012, PhD Thesis, Universität Köln, https://kups.ub. uni-koeln.de/4757/

Carone, L., \& Pätzold, M. 2007, Planet. Space Sci., 55, 643

Cassan, A., Kubas, D., Beaulieu, J.-P., et al. 2012, Nature, 481, 167

Chaplin, W. J., \& Miglio, A. 2013, ARA\&A, 51, 353

Chaplin, W. J., Basu, S., Huber, D., et al. 2014, ApJS, 210, 1

Claytor, Z. R., van Saders, J. L., Santos, A. R. G., et al. 2020, ApJ, 888, 43

Damiani, C., \& Díaz, R. F. 2016, A\&A, 589, A55

Degroote, P., Aerts, C., Baglin, A., et al. 2010, Nature, 464, 259

Dobbs-Dixon, I., Lin, D. N. C., \& Mardling, R. A. 2004, ApJ, 610, 464

Dziembowski, W. A., Goode, P. R., Pamyatnykh, A. A., \& Sienkiewicz, R. 1994, ApJ, 432, 417

Espinoza, N., Rackham, B. V., Jordán, A., et al. 2019, MNRAS, 482, 2065

Fleming, D. P., Barnes, R., Davenport, J. R. A., \& Luger, R. 2019, ApJ, 881, 88

Fressin, F., Torres, G., Charbonneau, D., et al. 2013, ApJ, 766, 81

Gallet, F., \& Bouvier, J. 2013, A\&A, 556, A36

Gallet, F., \& Bouvier, J. 2015, A\&A, 577, A98

Gänsicke, B. T., Schreiber, M. R., Toloza, O., et al. 2019, Nature, 576, 61

Goldreich, P., \& Keeley, D. A. 1977, ApJ, 211, 934

Guillot, T., Lin, D. N. C., Morel, P., Havel, M., \& Parmentier, V. 2014, in EAS Publications Series, EAS Publ. Ser., 65, 327

Hebb, L., Collier-Cameron, A., Triaud, A. H. M. J., et al. 2010, ApJ, 708, 224

Heller, R. 2019, A\&A, 628, A42

Hodžić, V., Triaud, A. H. M. J., Anderson, D. R., et al. 2018, MNRAS, 481, 5091

Hoppe, R., Bergemann, M., Bitsch, B., \& Serenelli, A. 2020, A\&A, 641, A73

Huber, D., Silva Aguirre, V., Matthews, J. M., et al. 2014, ApJS, 211, 2

Hut, P. 1980, A\&A, 92, 167

Hut, P. 1981, A\&A, 99, 126

Irwin, J., Hodgkin, S., Aigrain, S., et al. 2008, MNRAS, 384, 675

Jackson, B., Barnes, R., \& Greenberg, R. 2009, ApJ, 698, 1357

Kawaler, S. D. 1988, ApJ, 333, 236

Kunitomo, M., Guillot, T., Ida, S., \& Takeuchi, T. 2018, A\&A, 618, A132

Matt, S. P., Brun, A. S., Baraffe, I., Bouvier, J., \& Chabrier, G. 2015, ApJ, 799, L23

McCormac, J., Gillen, E., Jackman, J. A. G., et al. 2020, MNRAS, 493, 126

McQuillan, A., Mazeh, T., \& Aigrain, S. 2014, ApJS, 211, 24

Meibom, S., Barnes, S. A., Platais, I., et al. 2015, Nature, 517, 589

Meléndez, J., Bergemann, M., Cohen, J. G., et al. 2012, A\&A, 543, A29

Metcalfe, T. S., Creevey, O. L., Doğan, G., et al. 2014, ApJS, 214, 27

Metcalfe, T. S., Kochukhov, O., Ilyin, I. V., et al. 2019, ApJ, 887, L38

Murray, C. D., \& Dermott, S. F. 1999, Solar system dynamics (Cambridge, UK: Verlag Cambridge University Press)

Nelemans, G., Yungelson, L. R., Portegies Zwart, S. F., \& Verbunt, F. 2001, A\&A, 365, 491

Nielsen, M. B., Schunker, H., Gizon, L., \& Ball, W. H. 2015, A\&A, 582, A10

Nissen, P. E., Silva Aguirre, V., Christensen-Dalsgaard, J., et al. 2017, A\&A, 608, A112

Ogilvie, G. I., \& Lin, D. N. C. 2007, ApJ, 661, 1180

Oh, S., Price-Whelan, A. M., Brewer, J. M., et al. 2018, ApJ, 854, 138

Parker, E. N. 1958, ApJ, 128, 664

Petrovich, C., \& Muñoz, D. J. 2017, ApJ, 834, 116

Privitera, G., Meynet, G., Eggenberger, P., et al. 2016, A\&A, 591, A45

Queloz, D., Anderson, D. R., Collier Cameron, A., et al. 2010, A\&A, 517, L1

Rao, S., Meynet, G., Eggenberger, P., et al. 2018, A\&A, 618, A18

Rasio, F. A., Tout, C. A., Lubow, S. H., \& Livio, M. 1996, ApJ, 470, 1187

Sahlholdt, C. L., Feltzing, S., Lindegren, L., \& Church, R. P. 2019, MNRAS, 482,895

Santos, A. R. G., García, R. A., Mathur, S., et al. 2019, ApJS, 244, 21

Schatzman, E. 1962, Ann. Astrophys., 25, 18

Serenelli, A. M., Bergemann, M., Ruchti, G., \& Casagrande, L. 2013, MNRAS, 429, 3645

Serenelli, A., Johnson, J., Huber, D., et al. 2017, ApJS, 233, 23

Silva Aguirre, V., Davies, G. R., Basu, S., et al. 2015, MNRAS, 452, 2127

Skumanich, A. 1972, ApJ, 171, 565

Spina, L., Palla, F., Randich, S., et al. 2015, A\&A, 582, L6

Teske, J. K., Shectman, S. A., Vogt, S. S., et al. 2016, AJ, 152, 167

Van Eylen, V., Agentoft, C., Lundkvist, M. S., et al. 2018, MNRAS, 479, 4786

van Saders, J. L., Ceillier, T., Metcalfe, T. S., et al. 2016, Nature, 529, 181

Vanderburg, A., Johnson, J. A., Rappaport, S., et al. 2015, Nature, 526, 546

Veras, D., \& Fuller, J. 2019, MNRAS, 489, 2941

Weber, E. J., \& Davis, L., Jr. 1967, ApJ, 148, 217

Weiss, A., \& Schlattl, H. 2008, Ap\&SS, 316, 99

Weiss, L. M., Isaacson, H. T., Marcy, G. W., et al. 2018, AJ, 156, 254

Winn, J. N., Fabrycky, D., Albrecht, S., \& Johnson, J. A. 2010, ApJ, 718, L145

Yee, S. W., Winn, J. N., Knutson, H. A., et al. 2020, ApJ, 888, L5

Zahn, J. P. 1977, A\&A, 500, 121

Zhou, G., Huang, C. X., Bakos, G. Á., et al. 2019, AJ, 158, 141 